ARTICLE

Received 26 Mar 2014 | Accepted 13 Aug 2014 | Published 19 Sep $2014 \quad$ DOl: 10.1038/ncomms5983

\title{
Shoot-derived cytokinins systemically regulate root nodulation
}

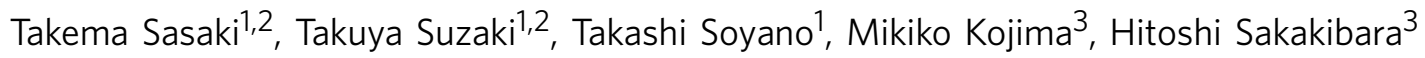

$\&$ Masayoshi Kawaguchi, ${ }^{1,2}$

Legumes establish symbiotic associations with nitrogen-fixing bacteria (rhizobia) in root nodules to obtain nitrogen. Legumes control nodule number through long-distance communication between roots and shoots, maintaining the proper symbiotic balance. Rhizobial infection triggers the production of mobile CLE-RS1/2 peptides in Lotus japonicus roots; the perception of the signal by receptor kinase HAR1 in shoots presumably induces the production of an unidentified shoot-derived inhibitor (SDI) that translocates to roots and blocks further nodule development. Here we show that, CLE-RS1/2-HAR1 signalling activates the production of shoot-derived cytokinins, which have an SDI-like capacity to systemically suppress nodulation. In addition, we show that LjIPT3 is involved in nodulation-related cytokinin production in shoots. The expression of LjIPT3 is activated in an HAR1-dependent manner. We further demonstrate shoot-to-root long-distance transport of cytokinin in L. japonicus seedlings. These findings add essential components to our understanding of how legumes control nodulation to balance nutritional requirements and energy status.

\footnotetext{
${ }^{1}$ Division of Symbiotic Systems, National Institute for Basic Biology, Nishigonaka 38, Myodaiji, Okazaki 444-8585, Japan. ${ }^{2}$ Department of Basic Biology in the School of Life Science of the Graduate University for Advanced Studies, Nishigonaka 38, Myodaiji, Okazaki 444-8585, Japan. ${ }^{3}$ Plant Productivity Systems Research Group, RIKEN Center for Sustainable Resource Science, 1-7-22, Suehiro, Tsurumi, Yokohama 230-0045, Japan. Correspondence and requests for materials should be addressed to M.Ka. (email: masayosi@nibb.ac.jp).
} 
T o establish symbiotic associations with rhizobia, a group of nitrogen-fixing bacteria, leguminous plants form nodules on their roots in response to rhizobial infection. The rhizobia colonize these nodules, supplying host plants with fixed atmospheric nitrogen while receiving photosyntates in turn. While such a symbiotic relationship generally is beneficial to both partners, the formation of excessive numbers of nodules inhibits the growth of the host plants. To avoid this effect, plants perform autoregulation of nodulation (AON), which systemically controls the number of nodules ${ }^{1}$. AON is a long-distance negativefeedback system involving root-shoot communication ${ }^{2-4}$. In the legume Lotus japonicus, two leucine-rich repeat receptor-like kinases, HYPERNODULATION ABERRANT ROOT FORMATION 1 (HAR1) and KLAVIER, have been identified as key components of AON that function in shoots ${ }^{5-9}$. The two proteins are orthologous to Arabidopsis CLAVATA1 and RECEPTOR-LIKE PROTEIN KINASE 2, respectively, which are involved in the maintenance of stem cell populations in shoot apical meristems via short-range cell-to-cell communication ${ }^{10,11}$. As an underlying mechanism of $\mathrm{AON}$, it has been postulated that signalling substances are produced in roots upon rhizobial infection, which then are transported to the $\operatorname{shoot}^{2,4}$. The perception of these primary signals in the shoot generates secondary signals. These shoot-derived signals, also called shootderived inhibitors (SDIs), are transported to the roots where they inhibit the initiation of new nodule development ${ }^{2,4,12-15}$. In L. japonicus, two peptides, CLE-ROOT SIGNAL 1 (CLE-RS1) and CLE-RS2, are strong candidates for root-derived mobile signalling molecules. Expression of the corresponding genes is induced specifically in infected roots, and CLE-RS2 glycopeptides are transported in the xylem to the shoot where they directly bind to HAR1 (refs 16,17). Application of arabinosylated CLE-RS peptides to shoots suppresses nodulation in an HAR1dependent manner ${ }^{17}$. Furthermore, the TOO MUCH LOVE (TML) F-box protein recently has been identified as a root-acting AON factor that inhibits nodulation downstream of HAR1 (refs 18,19). Although these results provide some insight into signalling mechanisms between the root and shoot, the mechanism and regulation of AON inhibition of nodule development remain mostly unclear. In particular, the identification of the SDI is lacking.

In this study, we focused on downstream events of the CLE-RS1/2-HAR1 signalling pathway. We show that the production of cytokinins (CKs) in shoots is activated by rhizobial infection, and that application of exogenous CKs to shoots can inhibit nodulation in a TML-dependent manner. Our results suggest that shoot-derived CKs systemically regulate root nodulation in AON.

\section{Results}

Phenotypic analyses and quantification of phytohormones. The har1 mutant shows a hypernodulation phenotype, possibly because of defects in SDI production ${ }^{4,8}$. On the other hand, rhizobial infection induces constitutive expression of CLE-RS1 or RS2, which encode peptides acting as ligands of HAR1, and inhibits further nodulation, probably due to increased SDI production in the shoot ${ }^{4,16,17}$. Consequently, we hypothesized
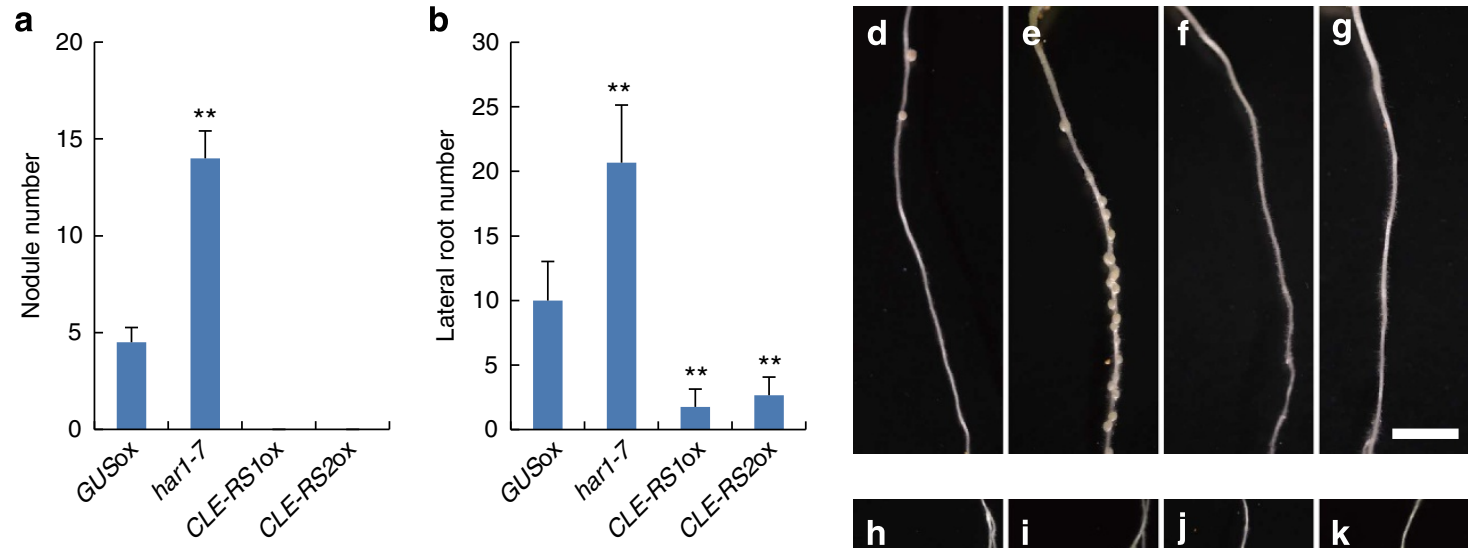

C
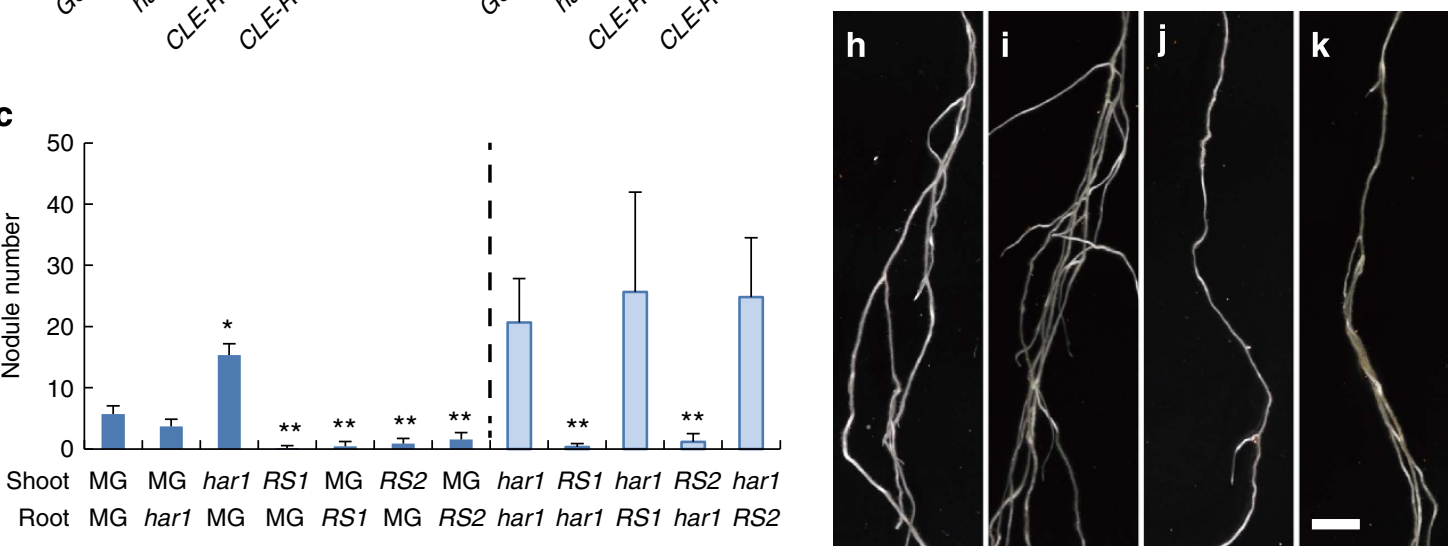

Figure 1 | Overexpression of CLE-RS1 and RS2 inhibit nodulation and lateral root formation. (a) Nodule number in GUSox, har1-7 mutant and CLE-RS1/20x plants $(n=6)$. Nodules were counted 14 days after infection with M. loti. (b) Lateral root numbers $(n=6-10)$ at 21 days after germination in the absence of rhizobia. (c) Shoot-to-root reciprocal grafting between MG-20, har1-7 mutant and CLE-RS1/2ox plants ( $n=6-9$ ). Nodules were counted 14 days after infection. Bars in (a-c) represent means \pm s.d. Asterisks indicate statistically significant differences from the control (a,b: GUSox, c: MG-20 or har1-7) at ${ }^{\star} P<0.05$ and ${ }^{\star \star} P<0.01$, according to the Student's $t$-test $(\mathbf{a}, \mathbf{b})$ and Tukey HSD (c). (d-k) Nodulation phenotypes (d-g) and root phenotypes (h-k) of GUSox (d,h), har1-7 mutant $(\mathbf{e}, \mathbf{i})$, CLE-RSTox $(\mathbf{f}, \mathbf{j})$ and RS2ox $(\mathbf{g}, \mathbf{k})$. Bars, $5 \mathrm{~mm}$ in $\mathbf{g}$ for $\mathbf{d}-\mathbf{g}, 1 \mathrm{~cm}$ in $\mathbf{k}$ for $\mathbf{h}-\mathbf{k}$. 
that SDI levels in shoots should differ between the harl mutant and the CLE-RS1/2 constitutive expression lines. If so, candidate SDIs should be identifiable by comparison of metabolite profiles between the different lines.

To this end, we generated stable $L$. japonicus transgenic plants in which either CLE-RS1 or RS2 were overexpressed under the control of the CaMV 35S promoter (CLE-RS1/2ox, Supplementary Fig. 1). As controls, plants expressing the GUS gene were generated (MG-20 background). Control plants (GUSox), har1-7 mutants, CLE-RS1ox and RS2ox plants were inoculated with Mesorhizobium loti, and nodules were counted 2 weeks after inoculation. har1-7 plants formed approximately three times more nodules than the control, whereas CLE-RSIox and RS2ox did not form nodules (Fig. 1a,d-g). This result was consistent with previous observations of nodulation inhibition by root-specific CLE-RS1 and RS2 expression following hairy root transformation ${ }^{16}$. In addition, we found that lateral root numbers in CLE-RS1ox and RS2ox were decreased to $18 \%$ and $27 \%$, respectively, of that in control plants (Fig. 1b,h,j,k). On the other hand, har1-7 plants formed approximately twice as many lateral roots as control plants (Fig. 1b,h,i). Thus, in terms of nodule and lateral root numbers, the CLE-RS1/2ox phenotypes were opposite to those of the har1-7 mutant. To see whether the nodulation inhibitory effects of CLE-RSI/2 overexpression are mediated through the shoots, we performed reciprocal grafting of shoots and roots between MG-20 (wild type), harl-7 mutants, CLE-RS1ox and RS2ox plants (Fig. 1c). Shoots of CLE-RS1ox and RS2ox inhibited nodulation in both wild-type and har1-7 mutant rootstocks, indicating that CLE-RS1 and RS2ox expression in the shoots is sufficient to block nodulation. Furthermore, the inhibition of nodulation in rootstocks overexpressing CLE-RS1/2 was suppressed by shoots of har1-7 mutants. These results confirmed that CLE-RS1/2 inhibited nodulation through shootroot communication in an HAR1-dependent manner, as observed previously ${ }^{16}$. Likewise, shoots from CLE-RS1ox plants inhibited lateral root formation in both wild-type and har1-7 mutant rootstocks (Supplementary Fig. 2), suggesting that lateral root formation was inhibited by CLE-RS1 expression in the shoot.

Recently, it was reported that SDI is a small amphiphilic compound (possiblemolecular mass $<10 \mathrm{kDa}$ ) unlikely to be a protein or an RNA molecule ${ }^{12-15}$. The harl mutant shows developmental alterations, including short primary roots and enhanced lateral root formation ${ }^{5}$. In contrast, the numbers of lateral roots were decreased in CLE-RS1ox and RS2ox plants (Fig. 1b). Therefore, we assumed that SDI might affect plant development in addition to its effects on nodule formation. Plant development is controlled by phytohormones, which are lowmolecular mass compounds ${ }^{15}$. Some phytohormones act as longdistance signals in the integrative regulation of development and in defence responses ${ }^{20-22}$. Thus it seemed the obvious next step to compare phytohormone compositions in MG-20 shoots with those of har1-7 mutant and CLE-RS1/2ox shoots. Given that SDI is produced downstream of CLE-RS1/2 and HAR1 (refs 2,16), we focused on phytohormones combining two trends, namely increased levels in shoots of both CLE-RS1ox and CLERS2ox plants, and decreased levels in har1-7 mutant shoots. In three independent analyses, only one of 39 compounds examined consistently showed both trends, namely iPRPs, which are intermediates of CK biosynthesis (Table 1; Supplementary Table 1). In addition, the amount of iPRPs in MG-20 shoots was significantly increased following rhizobial infection, reaching about twice the level detected in non-infected plants (Fig. 2). CKs are transferred from roots to shoots through the xylem ${ }^{23,24}$, and tZ-type CKs contribute the largest fraction of translocated $\mathrm{CKs}^{25}$. tZ-type CK levels in shoots were unaffected by rhizobial infection (Fig. 2). Thus, increased iPRPs levels in shoots probably are caused by CK synthesis rather than by CKs import from roots. In Arabidopsis, iPRP production is an initial step of CK biosynthesis, and controls the total amount of $\mathrm{CKs}^{26}$. Taken together, our results suggested that during nodulation, the production of CKs is induced in shoots through the activation of the CLE-RS1/2-HAR1 signalling pathway.

CK-feeding assay. To investigate whether CKs accumulating in shoots have SDI-like activities, we applied various concentrations of 6-benzylaminopurine (BAP) to MG-20, harl and tml seedlings via cut surfaces of cotyledons. Application of $10^{-6}$ and $10^{-5} \mathrm{M}$ BAP to MG-20 seedlings decreased the number of nodules to $44 \%$ and $13 \%$, respectively, compared with the buffer-only control treatment (Fig. 3a). Similarly, BAP inhibited nodulation in har1-7 seedlings in a dose-dependent manner (Fig. 3a). Evidently, CK applied to shoots inhibits the nodulation through a mechanism downstream of HAR1 action. The inhibitory effect was not observed in plants carrying the $t m l$ mutation (Fig. 3a), indicating that $T M L$, which functions in roots downstream of HAR1, is required for the inhibitory action of $\mathrm{CK}$.

We further examined effects of CK feeding on lateral root formation, which was affected in CLE-RS1/2ox, as well as on nodule formation (Fig. 1b). The formation of lateral roots was inhibited by BAP fed to shoots; lateral root numbers in MG-20 were decreased to $40 \%$ and $20 \%$ of the control by $10^{-7}$ and $10^{-6} \mathrm{M}$ BAP, respectively (Fig. 3b). The application of BAP to the shoot also inhibited lateral root formation in har1-7 mutants (Fig. 3b). tml mutants had similar lateral root numbers as MG-20, but the formation rate was not decreased by the BAP treatment (Fig. 3b), suggesting that shoot-derived CK controls lateral root formation as well as nodulation in a TML-dependent manner.

In Arabidopsis, the phloem has been demonstrated to be capable of transporting large amounts of $\mathrm{CKs}^{27}$. We applied labelled $\mathrm{CK}^{28}$ to establish whether CK fed to leaves is transported to roots in L. japonicus. In fact, the labelled CK soon was detected in root tips (Table 2; Supplementary Table 2). Evidently, CKs in the cotyledon are transported to the root tip in L. japonicas; thus it is likely that exogenously applied BAP (Fig. 3) was transported through the phloem to the root.

Expression analyses of $L$. japonicus IPT genes in shoots. Quantification of phytohormones had shown that CLE-RS1/20x shoots accumulated significantly larger amounts of iPRPs than MG-20 shoots. iPRPs are synthesized at initial steps of the CK biosynthetic pathway in a reaction catalysed by isopentenyltransferase (IPT). These steps are rate limiting in CK production, suggesting that production of iPRPs and CK levels are regulated by the expression of IPTs ${ }^{26,29}$. Six IPT genes $(L j I P T)$ can be found in the L. japonicus genomic database ${ }^{30}$. We examined the expression patterns of these $L j I P T$ genes of MG-20, har1-7 mutants and CLE-RS1/20x plants during nodulation (Fig. 4a,b; Supplementary Fig. 3). Among the five LjIPT genes for which activity could be detected, LjIPT1 and LjIPT3 showed increased expression in MG-20 shoots starting at 1 and 3 days, respectively, after inoculation. The other three $L j I P T$ genes showed no response (Supplementary Fig. 3). Responses of LjIPT1 and LjIPT3 genes were not observed in har1-7 shoots (Fig. 4a; Supplementary Fig. 3). Moreover, the expression of LjIPT3 was constitutively increased in CLE-RS1ox and RS2ox plants in the absence of rhizobia (Fig. 4b). The timing of the induction of LjIPT3 expression was consistent with the timing of the initiation of AON reported previously ${ }^{31}$. These findings suggested that the activation of LjIPT3 in the shoot is closely related to AON activation. 
Table 1 | Comparison of phytohormone levels between shoots of MG-20, har1-7 mutants and CLE-RS1/20x plants.

\begin{tabular}{|c|c|c|c|c|}
\hline & MG-20 & har1-7 & CLE-RS10X & CLE-RS2OX \\
\hline $\mathrm{tZ}$ & $0.70 \pm 0.42$ & $0.53 \pm 0.23$ & $1.12 \pm 0.55$ & ND \\
\hline $\mathrm{tZR}$ & $0.22 \pm 0.01$ & $0.15 \pm 0.01 \mathrm{dec}^{\star \star}$ & $0.26 \pm 0.04$ & $0.23 \pm 0.03$ \\
\hline tZRPs & $0.45 \pm 0.19$ & $0.42 \pm 0.10$ & $1.04 \pm 0.20 \mathrm{inc}^{\star}$ & $0.64 \pm 0.13$ \\
\hline DZRPs & $0.55 \pm 0.09$ & $0.48 \pm 0.15$ & $0.25 \pm 0.03 \mathrm{dec}^{\star}$ & $0.36 \pm 0.04$ \\
\hline iP & $0.47 \pm 0.34$ & $0.48 \pm 0.37$ & $1.20 \pm 0.95$ & $0.92 \pm 0.74$ \\
\hline iPR & $0.15 \pm 0.08$ & $0.09 \pm 0.04$ & $0.36 \pm 0.19$ & $0.25 \pm 0.12$ \\
\hline tZROG & $0.15 \pm 0.07$ & $0.07 \pm 0.04$ & $0.24 \pm 0.08$ & $0.09 \pm 0.03$ \\
\hline cZROG & $0.16 \pm 0.06$ & $0.08 \pm 0.03$ & $0.12 \pm 0.08$ & $0.23 \pm 0.18$ \\
\hline DZ9G & $0.87 \pm 0.01$ & $1.07 \pm 0.12$ & $0.42 \pm 0.10 \mathrm{dec}^{\star}$ & $0.48 \pm 0.21$ \\
\hline ¡P9G & $0.54 \pm 0.13$ & $0.37 \pm 0.08$ & $1.05 \pm 0.33$ & $0.69 \pm 0.15$ \\
\hline GA1 & $2.24 \pm 0.96$ & $1.34 \pm 0.35$ & $0.84 \pm 0.15$ & $2.21 \pm 0.72$ \\
\hline GA8 & $9.06 \pm 1.57$ & $6.31 \pm 0.80$ & $7.21 \pm 0.74$ & $7.42 \pm 1.91$ \\
\hline SA & $2,077.71 \pm 227.77$ & $4,083.6 \pm 838.25$ inc $^{\star}$ & $3,746.33 \pm 648.02$ inc $^{*}$ & $3,382.37 \pm 1429.06$ \\
\hline$J A$ & $104.12 \pm 1.42$ & $91.37 \pm 21.15$ & $93.29 \pm 47.40$ & $88.73 \pm 16.20$ \\
\hline IAA & $205.7 \pm 12.00$ & $331.64 \pm 146.73$ & $258.37 \pm 31.78$ & $321.73 \pm 107.61$ \\
\hline$A B A$ & $44.61 \pm 4.31$ & $43.44 \pm 4.72$ & $52.67 \pm 10.18$ & $51.76 \pm 13.60$ \\
\hline
\end{tabular}

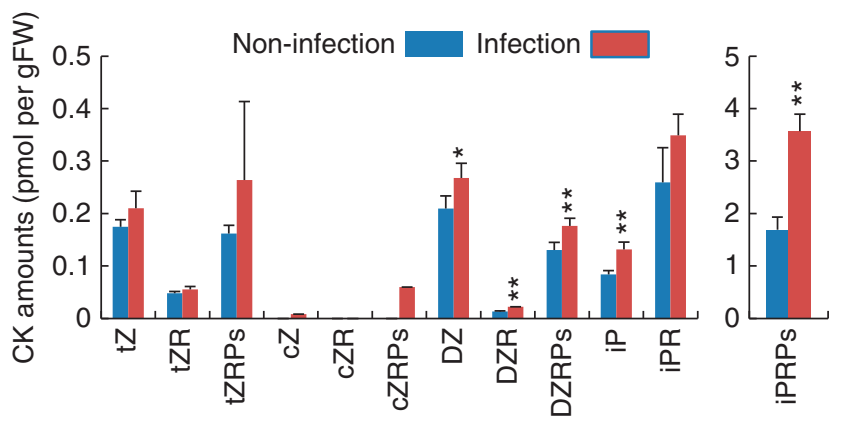

Figure 2 | iPRPs levels in shoots are increased by rhizobial infection.

CK levels in shoots were determined at 5 days after infection with $M$. loti (infection) or in the absence of rhizobia (non-infection). Means \pm s.d. from three independent experiments are shown. Asterisks indicate statistically significant differences between treatments $\left({ }^{\star} P<0.05,{ }^{\star \star} P<0.01\right.$;

Student's t-test).

We next examined the expression pattern of LjIPT3 in roots. Expression of LjIPT3 is induced in MG-20 roots by rhizobial infection, as shown recently (Fig. 4a) ${ }^{30}$. This infection-dependent activation was also observed in har1-7 (Fig. 4a), suggesting that, unlike in the case of shoots, HAR1 is not required for the activation of LjIPT3 in roots. In addition, the timing of LjIPT3 activation differs between shoots and roots; activation was observed 3 days after inoculation in shoots, but as early as 1 day after inoculation in roots (Fig. 4a).

We examined spatial expression patterns of LjIPT3 in shoots using transgenic ProLjIPT3:GUS plants, in which a $2.0-\mathrm{kb}$ fragment of the $L j I P T 3$ promoter region was inserted upstream of the GUS reporter gene. GUS signals were detected along a subset of leaf veins (Fig. 4c,d). Detailed analyses of leaf crosssections showed the signal restricted to phloem tissue (Fig. 4e,f).
This pattern resembled that of HAR1, which had been reported previously $^{32}$.

Functional analysis of the LjIPT3 gene. To elucidate the function of LjIPT3 in AON, we identified mutant lines with LORE1 retrotransposon inserts in the coding sequence of LjIPT3

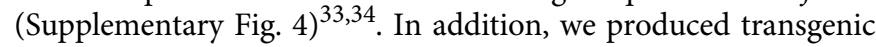
plants overexpressing LjIPT3 under the control of the CaMV 35S promoter (LjIPT3ox; Supplementary Fig. 1). In LjIPT3 shoots, iPRPs levels were five times higher than in MG-20 shoots in the absence of rhizobia (Supplementary Fig. 5), suggesting that overexpression of LjIPT3 is sufficient to increase iPRPs levels. LjIPT3ox lines and Ljipt3 mutants exhibited opposite phenotypes in terms of nodule number. LjIPT3ox lines formed only 30-40\% of the nodule number found in MG-20, whereas nodule numbers in Ljipt3 mutants exceeded those in Gifu B-129 by $58 \%$ (Fig. 5a-e). Evidently, LjIPT3 expression negatively influences nodulation. Furthermore, reciprocal grafting analysis showed that Ljipt3 shoots increased the number of nodules formed on Gifu B-129 rootstocks, while nodule numbers were comparable between Ljipt3 and Gifu B-129 rootstocks carrying Gifu B-129 shoots (Fig. 5f). These results demonstrated that LjIPT3 functions in the shoot-dependent inhibition of nodulation. The negative effect of $L j I P T 3$ expression in the shoot was confirmed by grafting LjIPT3ox shoots onto MG-20 wild-type rootstocks (Fig. 5g). As we also observed a reduction of nodule numbers in LjIPT3ox rootstocks carrying MG-20 shoots, the expression of LjIPT3 in roots appeared to inhibit nodulation as well (Fig. 5g).

Site of AON action in the root. To identify potential sites of AON action in the root, we examined the interaction between CLE-RS and CK signalling involving LHK1. Proper nodule development requires $L H K 1$, which encodes a putative CK receptor ${ }^{35,36}$. Initially, we performed grafting using rootstocks of 
a

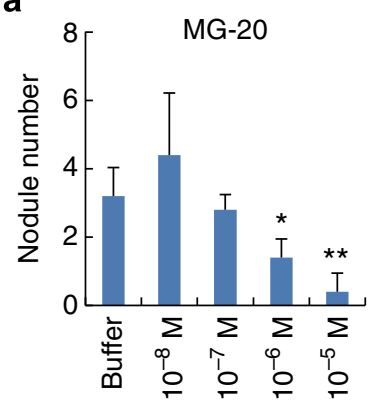

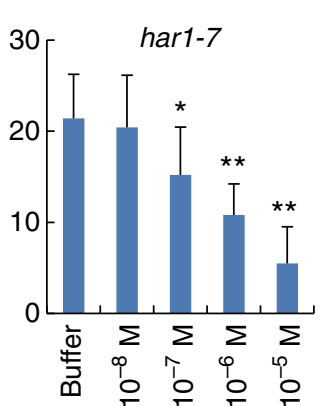
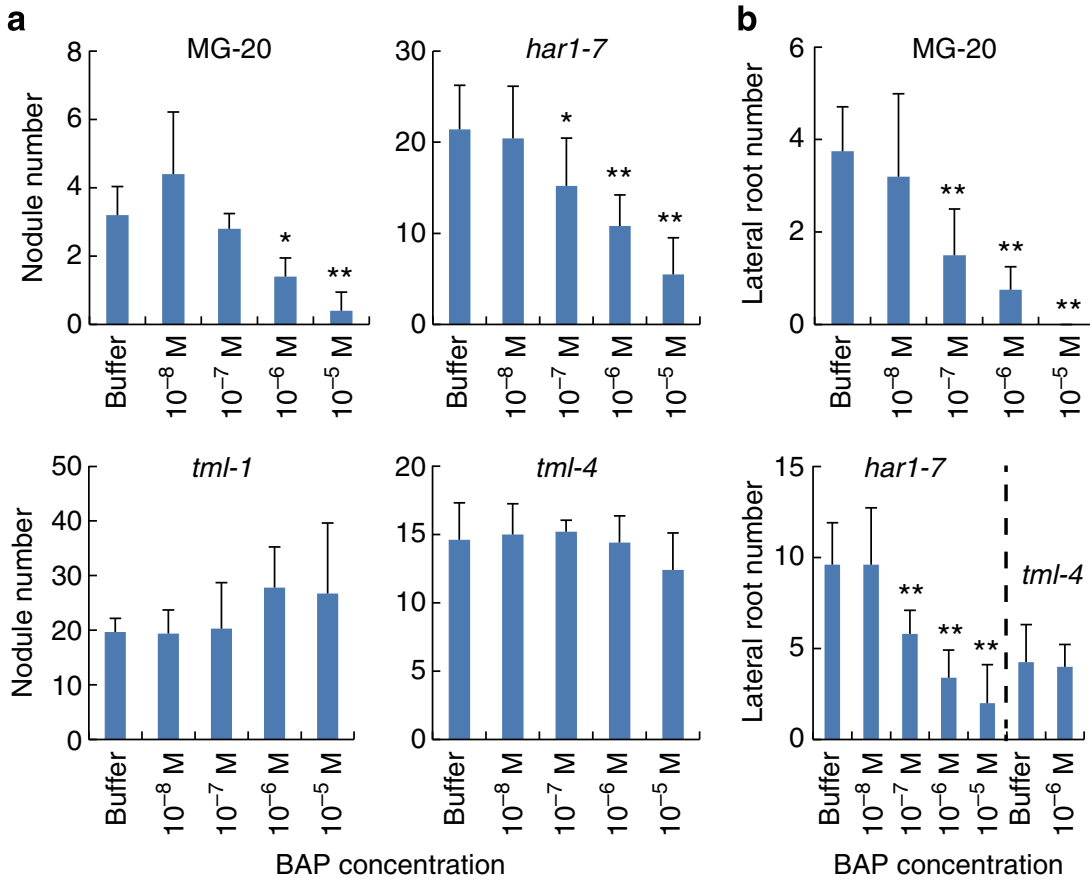

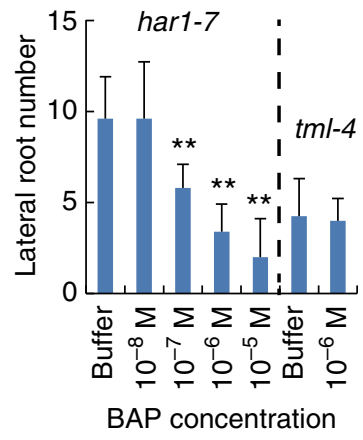

Figure 3 | BAP suppresses nodulation and lateral root formation in a TML-dependent manner. (a) Effects on nodulation of BAP application to shoots in MG-20 and har1-7, tml-1 and tml-4 mutants $(n=5)$. Distal halves of cotyledons were removed at 2 days after germination, and BAP was fed to the cut surfaces. Plants were inoculated with $M$. loti 1 day after starting BAP application. Nodules were counted 14 days after infection. (b) Effects on lateral root formation of BAP application to shoots in MG-20, har1-7 and tml-4 mutants $(n=5)$. Lateral roots were counted 21 days after starting BAP application in the absence of rhizobia. Data presented are means \pm s.d. Asterisks indicate statistically significant differences at ${ }^{\star} P<0.05$ and ${ }^{\star \star} P<0.01$, according to the Student's t-test.

Table 2 | Labelled CK applied to cotyledons is transported to the root tip.

Control 1 Control 2 Control 3 Sample 1 (pmol per 1sample) Sample 2 (pmol per 1sample) Sample 3 (pmol per sample)

Labelled iP, iPR and iPRPs

Labelled $t Z, t Z R$ and $t Z R P s$

Labelled $c Z$, tZR and cZRPs
ND ND

ND

ND
ND

ND

ND
1.0448
0.0237

0.0081
0.6634

0.0037
1.8270

0.0341

0.0066

CK, cytokinin; ND, not detected.

Labelled iPR, $\left.{ }^{13} C_{10}{ }_{15}^{15} N_{5}\right] N^{6}-\left(\Delta^{2}\right.$-isopentyl) adenine riboside, was applied to cut edges of cotyledons (3-day-old seedlings). Root tips ( $2 \mathrm{~mm}$ ) were collected $4 \mathrm{~h}$ after the treatment. Labelled and authentic CKs were analysed by mass spectrometry. The applied iPR is metabolized to iP, iPRPs, tZ-type CKs and cZ-type CKs. CK contents of untreated plants were determined as control. Data from three independent replicates are shown.

hit1 and snf2 mutants, which are loss-of- and gain-of-function mutants of $L H K 1$, respectively. It seems that the hit1 mutation reduces the number of nodules due to defective $\mathrm{CK}$ signalling in roots ${ }^{35}$, while the $s n f 2$ mutation causes the formation of so-called spontaneous nodules in the absence of rhizobia ${ }^{36}$. Grafting analysis showed that CLE-RS1ox shoots inhibited nodulation in hit1-1 and spontaneous nodulation in snf2 rootstocks (Supplementary Fig. 6). These results suggested that the site of AON action in roots may be downstream of LHK1. In Medicago truncatula, CK signalling involving MtCRE1, a counterpart of LHK1, may be involved in nodulation by mediating NSP2 expression $^{37}$. Therefore, we investigated the relationship between NSP2 expression and CLE-RS1/2 overexpression. In CLE-RS1ox and RS2ox plants, NSP2 expression was downregulated to $15 \%$ and $40 \%$, respectively, of that in control plants (MG-20) (Supplementary Fig. 7). Thus, AON seems to suppress the expression of NSP2 downstream of LHK1.

\section{Discussion}

AON systemically regulates the number of root nodules through shoot-root communication ${ }^{2,4}$. The inhibition of the formation of excess root nodules by a shoot-derived signal is a key element of AON, but molecular mechanisms involved in the production of the shoot-derived signal are poorly understood. We have shown that CLE-RS1/2-HAR1 signalling activates CK production in shoots, and that the synthesized CKs inhibit nodulation. TML, a component of $\mathrm{AON}$ acting in roots, is required for the inhibitory action of CKs. Thus, these results suggest that the inhibition of nodulation caused by CKs is under the control of AON especially between HAR1 and TML (Fig. 6). Our data also indicate that LjIPT3 is involved in CK production during nodulation. LjIPT3 expression is activated in shoots in an HAR1-dependent manner. Genetic analysis indicates that LjIPT3 acts as a negative regulator of nodulation. Since the nodulation phenotype of Ljipt3 knockout mutants is milder than that of canonical AON mutants, several $L j I P T$ genes may function redundantly in the control of nodulation. Generally, Arabidopsis IPT genes seem to have overlapping functions in diverse processes of plant development ${ }^{38}$. Promoter-reporter analyses showed that the LjIPT3 promoter was active specifically in phloem cells, suggesting that CKs may be synthesized in the shoot phloem upon rhizobial infection of roots (Fig. 6). In Arabidopsis, iP-type CKs are transported from shoot to root via phloem sieve tubes $^{25,39}$, and we demonstrated that CKs are transported from 
a
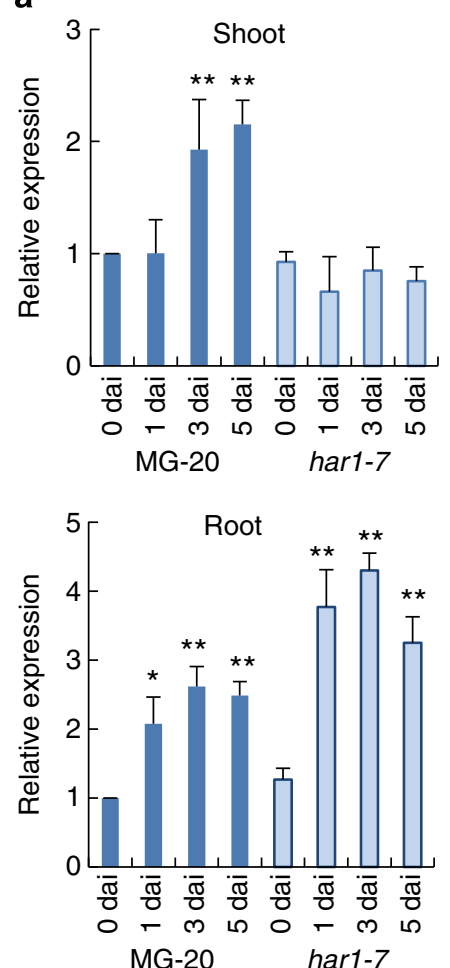

b

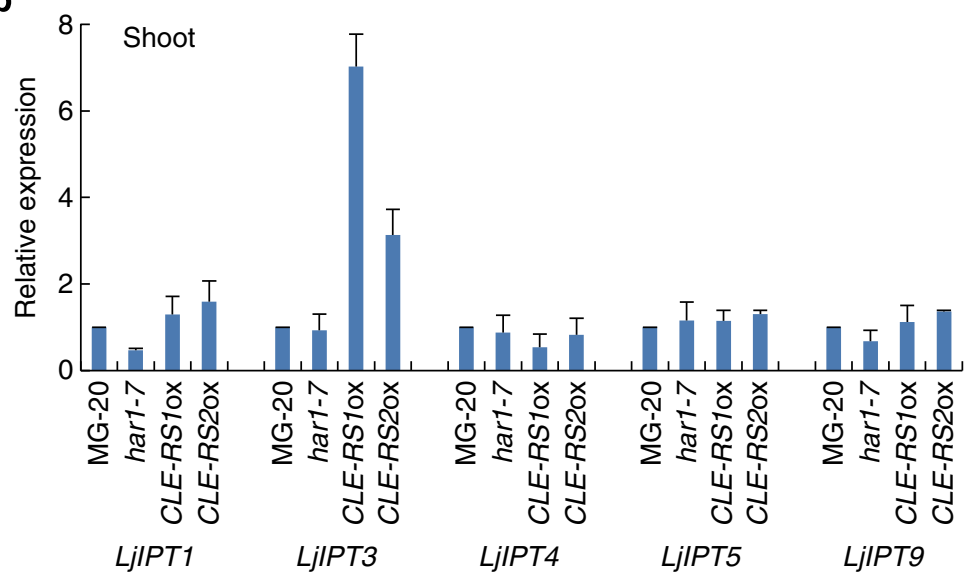

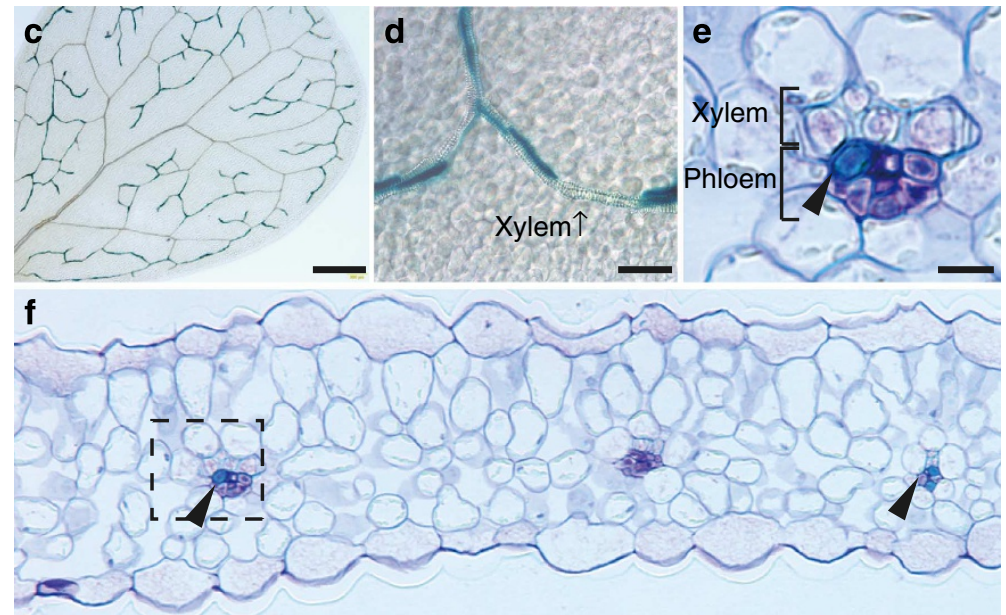

Figure 4 | Expression analyses of LjIPTgenes. (a) Relative expression of LjIPT3 genes in shoots and roots of MG-20 and har1-7 plants at different times after infection with $M$. loti (dai). Dai, days after infection. Asterisks indicate statistically significant differences at ${ }^{\star} P<0.05$ and ${ }^{\star \star} P<0.01$, according to the Student's t-test. (b) Relative expression of LjIPTs genes in shoots of various plant lines grown in the absence of rhizobia. Expression of $L j I P T 2$ was not detected. $(\mathbf{a}, \mathbf{b})$ Data presented are means \pm s.d. of three biological repeats. Transcript amounts in different samples were normalized to those of ubiquitin. (c,d) GUS staining in leaves of ProLjIPT3:GUS plants at 3 days after infection with M. loti. (e,f) Cross-section of a leaf stained with 0.05\% toluidine blue. The arrowhead highlights an area showing the GUS signal. (e) Magnified image of the boxed region in $\mathbf{f}$. Bars, $0.5 \mathrm{~mm}(\mathbf{c}), 50 \mu \mathrm{m}(\mathbf{d}, \mathbf{f}), 10 \mu \mathrm{m}(\mathbf{e})$.

cotyledons to root tips in L. japonicus. In addition, in our grafting experiments nodulation was inhibited when LjIPT3ox plants were used as scions (Fig. 5g), consistent with the notion that shoot-derived CKs inhibit nodulation in roots.

CKs have long been suggested to act as positive regulators of nodule development. For example, a nodulation-deficient Rhizobium mutant could be recovered by introducing a gene related to trans-zeatin secretion ${ }^{40}$, and recent genetic analyses demonstrated that activation of CK signalling in host plants is necessary and sufficient to form nodule primordia ${ }^{35,36,41}$. Moreover, LjIPT3 is involved in CK production in roots with positive effects on nodulation ${ }^{30}$. In contrast, our results showed a negative effect of shoot-derived CKs on nodulation, as CKs act systemically to restrict the number of nodules. The production of CKs in both shoots and roots during nodulation is induced by expression of LjIPT3, but the timing of LjIPT3 activation is different between shoots and roots. In addition, our data also indicated that $L j I P T 3$ expression in shoots is induced in an HAR1-dependent manner, whereas HAR1 is not required for the activation of LjIPT3 in roots. These findings suggest that CKs, which negatively affect nodulation, may be produced following AON signalling in shoots, whereas CKs with positive effects on nodulation may be produced independently of AON signalling in roots. Therefore, different induction mechanisms including differential timing of CK production and HAR1 dependence can provide CKs with a dual role in nodulation. The visible loss-offunction phenotypes of LjIPT3 might differ drastically between different lines due to the dual role of CKs. RNA interference knockdown lines of LjIPT3 decrease nodule number, probably because the nodulation-promoting function of LjIPT3 is predominantly affected in this case ${ }^{30}$. In contrast, knockout lines used in the present study (Ljipt3-1 and Ljipt3-2) showed increased nodule numbers, presumably because of defects in the nodulation-restricting role of LjIPT3 in shoots. In this case, any inhibition of the positive effects of LjIPT3 in roots does not become apparent. Our grafting experiment and expression analysis suggested that AON may suppress NSP2 expression downstream of LHK1. CK application to roots significantly downregulates NSP2 expression after a transient upregulation ${ }^{37}$. Similarly, shoot-derived CKs may control the number of nodules by downregulating NSP2 expression. Our grafting experiment also showed that AON retains its inhibitory effects on nodulation in the LHK1 mutant background. L. japonicus has three putative CKs receptors with overlapping functions in the control of nodulation ${ }^{42}$. On the basis of these findings and our present results, we hypothesize that $\mathrm{CKs}$ with negative effects on nodulation may suppress the expression of NSP2 via CK receptor(s) other than LHK1. Such differential function of CK 


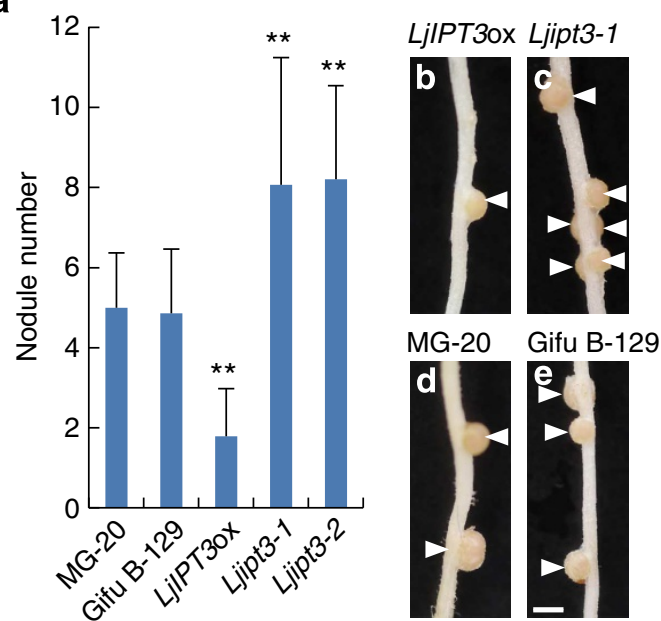

f

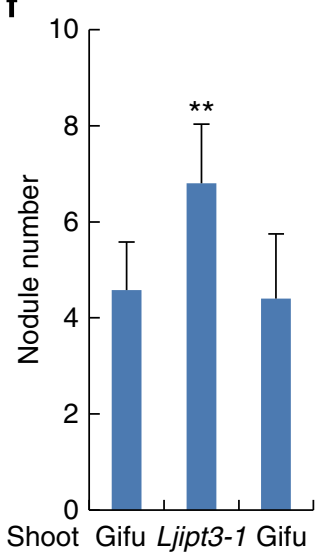

Root Gifu Gifu Ljipt3-1 g

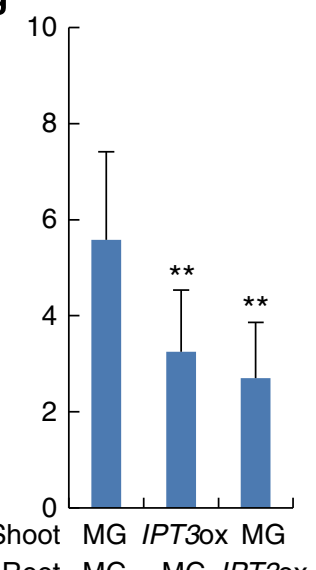

Figure 5 | LjIPT3 effects on nodule number. (a) Nodule number of wild type (MG-20 and Gifu B-129), LjIPT3ox plants (MG-20 background) and Ljipt3 mutants (Gifu B-129 background) $(n=14-15)$. (b-e) Nodule phenotypes, arrowheads indicate nodules. $(\mathbf{f}, \mathbf{g})$ Nodule numbers in shoot-to-root reciprocal grafts between Gifu B-129 and Ljipt3-1 mutants $(n=10-12)(\mathbf{f})$ and between MG-20 and LjIPT3ox $(n=10-12)(\mathbf{g})$. Nodules were counted 21 days after infection with $M$. loti. Data presented are means \pm s.d. Asterisks indicate statistically significant differences at ${ }^{\star \star} P<0.01$ according to the Student's $t$-test. Bar, $1 \mathrm{~mm}$.

receptors may explain the dual role of CKs. On the other hand, we cannot rule out the possibility that additional components induced downstream of CK production in shoots act systemically to inhibit nodulation.

We showed that CKs produced in shoots block the formation of lateral roots in a TML-dependent manner. This implies that nodulation and lateral root formation are systemically regulated via the same pathway downstream of CK production in the shoot. Although tml-4 mutants were insensitive to shoot-applied CKs with regard to the inhibition of lateral root formation, lateral root numbers of the mutants were similar to that of the wild type. This suggests that the TML regulation of lateral root development depends on additional factors. For instance, nitrate influences both lateral root development and nodulation ${ }^{43-45}$. Nodulation is suppressed by nitrate, and AON factors such as HAR1, KLV and TML are involved in this inhibitory regulation ${ }^{1,2,5,6,18}$. Nitrate systemically regulates root architecture, and induces IPT3 expression in Arabidopsis shoots ${ }^{44,46,47}$. Thus, CKs may play a central role in the systemic regulation of root lateral organ development. However, the underlying mechanisms remain

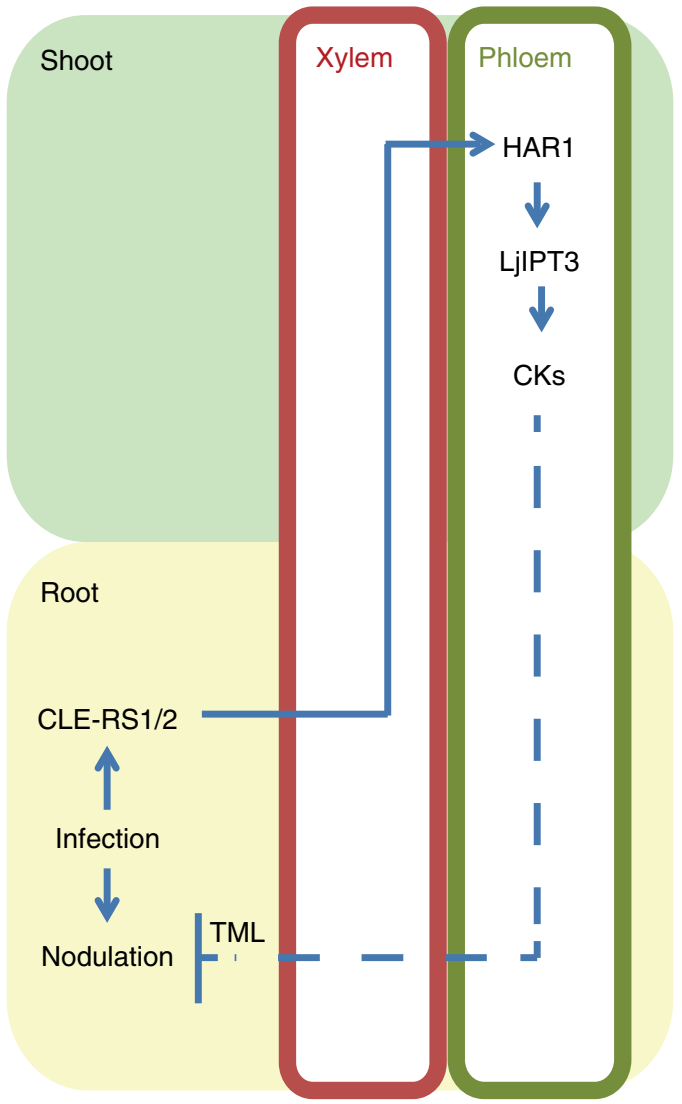

Figure 6 | Schematic illustration of the proposed AON model. Demonstrated and putative regulatory relationships are indicated by bold and dotted lines, respectively. See text for details.

obscure. KISS ME DEADLY, a Kelch repeat F-box protein that resembles TML, acts as a negative regulator of $\mathrm{CK}$ signalling in Arabidopsis ${ }^{19,48}$. Thus, interactions between CKs and TML may be essential for the negative regulation of nodulation, as well as lateral root development by CKs. Further investigations focusing on the interaction between CKs and TML will elucidate how CKs achieve their dual function in the control of nodule development, and may give insights into the long-distance regulatory mechanism required for nodulation and lateral root development.

\section{Methods}

Plant materials and growth conditions. We used L. japonicus accessions Miyakojima MG-20 and Gifu B-129 as the wild type, and the Ljipt3-1 and Lipt3-2 LORE1 retrotransposon insertion lines with plant IDs 30001893 and 30012123 , respectively. Hypernodulation mutants used were harl-7 (ref. 18), tml-1 and tml-4 (ref. 19). Mutants of LHK1 were hit1-1 (ref. 35) and snf2 (ref. 9). Plants were grown in autoclaved vermiculite supplemented with Broughton and Dilworth (B\&D) solution ${ }^{49}$ with $0.5 \mathrm{mM} \mathrm{KNO}_{3}$ under $16 \mathrm{~h}$ light $/ 8 \mathrm{~h}$ dark cycles. The rhizobium strain Mesorhizobium loti MAFF 303099 was used for nodulation.

Constructs and transformation. p35S-CLE-RS1, p35S-CLE-RS2 and p35S-GUS constructs reported previously ${ }^{16}$ were used to produce stably transformed plants that constitutively expressed either $C L E-R S 1$ or $C L E-R S 2$. For overexpressing $L j I P T 3$, the $L j I P T 3$ open-reading frame fragment was amplified from MG-20 genomic DNA using the specific primer set $\left(5^{\prime}\right.$-CACCGATCAGATACCAAT TTTGCA- $3^{\prime}$ and $5^{\prime}$-ACCACCCTCTATGAACATAACTAAC- $3^{\prime}$ ), and cloned into the pENTR/D-TOPO vector (Invitrogen). The insert was placed downstream of the p35S promoter of $\mathrm{pH} 7 \mathrm{WG} 2 \mathrm{D}^{50}$ by LR clonase (Invitrogen). For promoter analysis of $L j I P T 3$, the 2-kb DNA fragment upstream of the putative translation initiation codon was cloned into the pENTR/D-TOPO vector and transferred to pGWB3 (ref. 51) by the Gateway LR reaction. The promoter fragment was amplified from MG-20 genomic DNA using the specific primer set (5'-CACCTTTGGTAATTGA ATTTAATGGGCA- $3^{\prime}$ and $5^{\prime}$-GGTGAATTGCAAAATTGGTATCTGATC- $3^{\prime}$ ). Resultant binary vectors were introduced into Agrobacterium tumefaciens strain AGL1 and transformed into MG-20 as described previously ${ }^{52}$. 
Grafting experiments. Seeds were sown on vertical $1 \%$ agar plates and grown for 2 days in darkness. Plants were further cultured for 2 days in $16 \mathrm{~h}$ light per $8 \mathrm{~h}$ dark cycles. Rootstocks were produced by cutting off the hypocotyl at its base. A short vertical slit $(\sim 2 \mathrm{~mm})$ was made in the basal hypocotyl stump with a scalpel blade. A shoot scion was inserted into this vertical slit. Grafted plants were grown on filter papers saturated with sterilized water in Petri dishes for 4 days, before transfer to vermiculite supplemented with $\mathrm{B} \& \mathrm{D}$ solution and $0.5 \mathrm{mM} \mathrm{KNO}_{3}$ (ref. 18).

Quantification of phytohormones. Shoots of 2-week-old plants (about $100 \mathrm{mg}$ fresh weight) were collected 3 days after inoculation with $M$. loti, frozen in liquid $\mathrm{N}_{2}$ and stored at $-80^{\circ} \mathrm{C}$. Frozen shoots were crushed using a TissueLyser (Qiagen Retsch $\mathrm{GmbH}$ ) with a zirconia bead (diameter, $5 \mathrm{~mm}$ ) in a 2-ml microcentrifuge tube, and determination of primary metabolites was conducted according to Kojima et al. ${ }^{53}$ In brief, the extract was passed through an Oasis HLB column (Waters), and the hormone-containing fraction was passed through an Oasis MCX column (Waters). Each of the phytohormones were eluted with methanol (elution 1), $0.35 \mathrm{M}$ ammonia (elution 2) and $0.35 \mathrm{M}$ ammonia in $60 \%(\mathrm{v} / \mathrm{v})$ methanol (elution 3). The elution 1 fraction was passed through a DEAE-cellulose column (Vivapure D Mini M, Vivascience). Phytohormones were measured using a liquid chromatography-tandem mass chromatography system (ACQUITY UPLCSystem/ Quattro Ultima Pt; Waters) with an ODS column (ACQUITY UPLC BEH C ${ }_{18}$, Waters). Determined phytohormones were trans-zeatin (tZ); $\mathrm{tZ}$ riboside (tZR); tZR $5^{\prime}$-phosphates (tZRPs); cis-zeatin (cZ); cZ riboside (cZR); cZR $5^{\prime}$-phosphates (cZRPs); dihydrozeatin (DZ); DZ riboside (DZR); DZ riboside $5^{\prime}$-phosphates (DZRPs); $N^{6}-\left(\Delta^{2}\right.$-isopentenyl) adenine (iP); iP riboside (iPR); iPR $5^{\prime}$-phosphates (iPRPs); tZ-7-N-glucoside (tZ7G); tZ-9-N-glucoside (tZ9G); tZ-O-glucoside (tZOG); cZ-O-glucoside (cZOG); tZR-O-glucoside (tZROG); cZR-O-glucoside (cZROG); tZRPs-O-glucoside (tZRPsOG); cZRPs-O-glucoside (cZRPsOG); DZ-9-N-glucoside (DZ9G); iP-7-N-glucoside (iP7G); iP-9-N-glucoside (iP9G); gibberellin (GA); salicylic acid (SA); jasmonic acid (JA); indole-3-acetic acid (IAA); abscisic acid (ABA)

Quantification of CKs. Shoots of 2-week-old plants (about $100 \mathrm{mg}$ fresh weight) were collected 5 days after inoculation with $M$. loti (infection treatment) or application of B\&D solution in the absence of rhizobia (non-infection treatment), frozen in liquid $\mathrm{N}_{2}$ and stored at $-80^{\circ} \mathrm{C}$. Frozen shoots were crushed using a TissueLyser with a zirconia bead in a $2-\mathrm{ml}$ microcentrifuge tube, and determination of CK contents was conducted according to Kojima et al. ${ }^{53}$ The CK quantification method was the same as the phytohormones quantification method described above.

CK-feeding assay. The distal half of a cotyledon was removed from 2-day-old seedlings grown on vermiculate. The remaining cotyledon stump was inserted into a plastic tube filled with $10 \mathrm{mM}$ MES ( $\mathrm{pH}$ 6.2) containing BAP. On the next day, plants were inoculated with rhizobia. The number of nodules was counted 2 weeks after inoculation.

Expression analysis. Total RNA was isolated from plants using the Concert Plant RNA Reagent (Invitrogen). First-strand complementary DNA was synthesized using the QuantiTect Reverse Transcription Kit (Qiagen). Real-time reverse transcriptase-PCR was performed on an ABI prism 7000 sequence detection system (Applied Biosystems) using the THUNDERBIRD SYBR qPCR Mix (Toyobo) according to the manufacturer's protocol. The primers used are listed in Supplementary Table 3. Expression of ubiquitin served as the reference.

GUS assay. Leaves were incubated in $90 \%$ acetone for $15 \mathrm{~min}$ at $-20{ }^{\circ} \mathrm{C}$, and then incubated with GUS-staining buffer $\left(0.5 \mathrm{mg} \mathrm{ml}^{-1} \mathrm{X}\right.$-gluc, $100 \mathrm{mM}$ phosphate buffer $)^{54}$ for $24 \mathrm{~h}$ at $37^{\circ} \mathrm{C}$. The samples were washed twice with $100 \mathrm{mM} \mathrm{NaPO}_{4}$ ( $\mathrm{pH} 7.0$ ), bleached with $70 \%$ ethanol at room temperature and dehydrated in $100 \%$ ethanol. Then the samples were embedded in Technovit 7100 resin (Haraeus Kulzer) according to the manufacturer's protocol and sectioned (10 $\mu \mathrm{m}$ thickness) using a Microtome RM2255 (Leica). The sections were stained with $0.02 \%$ toluidine blue (Sigma) before observation.

CK transport assay. The distal half of each cotyledon was excised from seedlings grown for 3 days on $1 \%$ agar plates. Three $\mu$ of $0.5 \%$ agar containing $100 \mathrm{pmol}$ isotope-labelled iPR, $\left[{ }^{13} \mathrm{C}_{10},{ }^{15} \mathrm{~N}_{5}\right] \mathrm{N}^{6}-\left(\Delta^{2}\right.$-isopentyl) adenine riboside (Sakakibara laboratory, RIKEN Center for Sustainable Resource Science $)^{28}$, were put on the cut surface of each cotyledon. Plants were placed vertically on sterilized filter paper saturated with sterilized water in Petri dishes for $4 \mathrm{~h}$. Twenty root tips $(2 \mathrm{~mm})$ were collected and stored at $-80^{\circ} \mathrm{C}$. Frozen root tips were crushed using a TissueLyser with a zirconia bead in a $2-\mathrm{ml}$ microcentrifuge tube and CK contents were quantified as previously described ${ }^{53}$. The method was the same as the phytohormones quantification method described above.

\section{References}

1. Caetano-Anolles, G. \& Gresshoff, P. M. Plant genetic control of nodulation. Annu. Rev. Microbiol. 45, 345-382 (1991).

2. Oka-Kira, E. \& Kawaguchi, M. Long-distance signaling to control root nodule number. Curr. Opin. Plant Biol. 9, 496-502 (2006).

3. Magori, S. \& Kawaguchi, M. Long-distance control of nodulation: molecules and models. Mol. Cells 27, 129-134 (2009).

4. Ferguson, B. J. et al. Molecular analysis of legume nodule development and autoregulation. J. Integr. Plant Biol. 52, 61-76 (2010).

5. Wopereis, J. et al. Short root mutant of Lotus japonicus with a dramatically altered symbiotic phenotype. Plant J. 23, 97-114 (2000).

6. Oka-Kira, E. et al. klavier (klv), a novel hypernodulation mutant of Lotus japonicus affected in vascular tissue organization and floral induction. Plant $J$. 44, 505-515 (2005).

7. Krusell, L. et al. Shoot control of root development and nodulation is mediated by a receptor-like kinase. Nature 420, 422-426 (2002).

8. Nishimura, R. et al. HAR1 mediates systemic regulation of symbiotic organ development. Nature 420, 426-429 (2002).

9. Miyazawa, H. et al. The receptor-like kinase KLAVIER mediates systemic regulation of nodulation and non-symbiotic shoot development in Lotus japonicus. Development 137, 4317-4325 (2010).

10. Clark, S. E., Williams, R. W. \& Meyerowitz, E. M. The CLAVATA1 gene encodes a putative receptor kinase that controls shoot and floral meristem size in Arabidopsis. Cell 89, 575-585 (1997).

11. Kinoshita, A. et al. RPK2 is an essential receptor-like kinase that transmits the CLV3 signal in Arabidopsis. Development 137, 3911-3920 (2010).

12. Kenjo, T., Yamaya, H. \& Arima, Y. Shoot-synthesized nodulation-restricting substances of wild-type soybean present in two different high-performance liquid chromatography peaks of the ethanol-soluble medium-polarity fraction. Soil Sci. Plant Nutr. 56, 399-406 (2010).

13. Yamaya, H. \& Arima, Y. Evidence that a shoot-derived substance is involved in regulation of the super-nodulation trait in soybean. Soil Sci. Plant Nutr. 56, 115-122 (2010).

14. Yamaya, H. \& Arima, Y. Shoot-synthesized nodulation-restricting substances are present in the medium-polarity fraction of shoot extracts from wild-type soybean plants. Soil Sci. Plant Nutr. 56, 418-421 (2010).

15. Lin, Y. H., Ferguson, B. J., Kereszt, A. \& Gresshoff, P. M. Suppression of hypernodulation in soybean by a leaf-extracted, NARK- and Nod factor-dependent, low molecular mass fraction. New Phytol. 185, 1074-1086 (2010).

16. Okamoto, S. et al. Nod factor/nitrate-induced CLE genes that drive HAR1-mediated systemic regulation of nodulation. Plant Cell Physiol. 50, 67-77 (2009)

17. Okamoto, S., Shinohara, H., Mori, T., Matsubayashi, Y. \& Kawaguchi, M. Root-derived CLE glycopeptides control nodulation by direct binding to HAR1 receptor kinase. Nat. Commun. 4, 2191 (2013).

18. Magori, S. et al. TOO MUCH LOVE, a root regulator associated with the long-distance control of nodulation in Lotus japonicus. Mol. Plant Microbe Interact. 22, 259-268 (2009).

19. Takahara, M. et al. TOO MUCH LOVE, a novel Kelch repeat-containing F-box protein, functions in the long-distance regulation of the legume-Rhizobium symbiosis. Plant Cell Physiol. 54, 433-447 (2013).

20. Cheng, X., Ruyter-Spira, C. \& Bouwmeester, H. The interaction between strigolactones and other plant hormones in the regulation of plant development. Front. Plant Sci. 4, 199 (2013).

21. Heil, M. \& Ton, J. Long-distance signalling in plant defence. Trends Plant Sci. 13, 264-272 (2008)

22. Kudo, T., Kiba, T. \& Sakakibara, H. Metabolism and long-distance translocation of cytokinins. J. Integr. Plant Biol. 52, 53-60 (2010).

23. Ko, D. et al. Arabidopsis ABCG14 is essential for the root-to-shoot translocation of cytokinin. Proc. Natl Acad. Sci. USA 111, 7150-7155 (2014).

24. Zhang, K. et al. Arabidopsis ABCG14 protein controls the acropetal translocation of root-synthesized cytokinins. Nat. Commun. 5, 3274 (2014).

25. Hirose, N. et al. Regulation of cytokinin biosynthesis, compartmentalization and translocation. J. Exp. Bot. 59, 75-83 (2008).

26. Miyawaki, K., Matsumoto-Kitano, M. \& Kakimoto, T. Expression of cytokinin biosynthetic isopentenyltransferase genes in Arabidopsis: tissue specificity and regulation by auxin, cytokinin, and nitrate. Plant J. 37, 128-138 (2004).

27. Bishopp, A. et al. Phloem-transported cytokinin regulates polar auxin transport and maintains vascular pattern in the root meristem. Curr. Biol. 21, 927-932 (2011).

28. Tokunaga, H. et al. Arabidopsis lonely guy (LOG) multiple mutants reveal a central role of the LOG-dependent pathway in cytokinin activation. Plant J. 69, 355-365 (2012).

29. Takei, K., Sakakibara, H. \& Sugiyama, T. Identification of genes encoding adenylate isopentenyltransferase, a cytokinin biosynthesis enzyme, in Arabidopsis thaliana. J. Biol. Chem. 276, 26405-26410 (2001). 
30. Chen, Y. et al. Knockdown of LjIPT3 influences nodule development in Lotus japonicus. Plant Cell Physiol. 55, 183-193 (2013).

31. Suzuki, A. et al. Split-root study of autoregulation of nodulation in the model legume Lotus japonicus. J. Plant Res. 121, 245-249 (2008).

32. Nontachaiyapoom, S. et al. Promoters of orthologous Glycine max and Lotus japonicus nodulation autoregulation genes interchangeably drive phloem-specific expression in transgenic plants. Mol. Plant Microbe Interact. 20, 769-780 (2007).

33. Urbanski, D. F., Malolepszy, A., Stougaard, J. \& Andersen, S. U. Genome-wide LORE1 retrotransposon mutagenesis and high-throughput insertion detection in Lotus japonicus. Plant J. 69, 731-741 (2012).

34. Fukai, E. et al. Establishment of a Lotus japonicus gene tagging population using the exon-targeting endogenous retrotransposon LORE1. Plant J. 69, 720-730 (2012)

35. Murray, J. D. et al. A cytokinin perception mutant colonized by Rhizobium in the absence of nodule organogenesis. Science 315, 101-104 (2007).

36. Tirichine, L. et al. A gain-of-function mutation in a cytokinin receptor triggers spontaneous root nodule organogenesis. Science 315, 104-107 (2007).

37. Ariel, F. et al. Two direct targets of cytokinin signaling regulate symbiotic nodulation in Medicago truncatula. Plant Cell 24, 3838-3852 (2012).

38. Miyawaki, K. et al. Roles of Arabidopsis ATP/ADP isopentenyltransferases and tRNA isopentenyltransferases in cytokinin biosynthesis. Proc. Natl Acad. Sci. USA 103, 16598-16603 (2006).

39. Sakakibara, H. Cytokinins: activity, biosynthesis, and translocation. Annu. Rev. Plant Biol. 57, 431-449 (2006).

40. Cooper, J. B. \& Long, S. R. Morphogenetic rescue of Rhizobium meliloti nodulation mutants by trans-zeatin secretion. Plant Cell 6, 215-225 (1994).

41. Heckmann, A. B. et al. Cytokinin induction of root nodule primordia in Lotus japonicus is regulated by a mechanism operating in the root cortex. Mol. Plant Microbe Interact. 24, 1385-1395 (2011).

42. Held, M. et al. Lotus japonicus cytokinin receptors work partially redundantly to mediate nodule formation. Plant Cell 26, 678-694 (2014).

43. Zhang, H., Jennings, A., Barlow, P. W. \& Forde, B. G. Dual pathways for regulation of root branching by nitrate. Proc. Natl Acad. Sci. USA 96, 6529-6534 (1999).

44. Alvarez, J. M., Vidal, E. A. \& Gutierrez, R. A. Integration of local and systemic signaling pathways for plant $\mathrm{N}$ responses. Curr. Opin. Plant Biol. 15, 185-191 (2012).

45. Cho, M. J. \& Harper, J. E. Effect of localized nitrate application on isoflavonoid concentration and nodulation in split-root systems of wild-type and nodulation-mutant soybean plants. Plant Physiol. 95, 1106-1112 (1991).

46. Ruffel, S. et al. Nitrogen economics of root foraging: transitive closure of the nitrate-cytokinin relay and distinct systemic signaling for $\mathrm{N}$ supply vs demand. Proc. Natl Acad. Sci. USA 108, 18524-18529 (2011).

47. Takei, K. et al. AtIPT3 is a key determinant of nitrate-dependent cytokinin biosynthesis in Arabidopsis. Plant Cell Physiol. 45, 1053-1062 (2004).

48. Kim, H. J., Chiang, Y. H., Kieber, J. J. \& Schaller, G. E. SCF(KMD) controls cytokinin signaling by regulating the degradation of type-B response regulators. Proc. Natl Acad. Sci. USA 110, 10028-10033 (2013).
49. Broughton, W. J. \& Dilworth, M. J. Control of leghaemoglobin synthesis in snake beans. Biochem. J. 125, 1075-1080 (1971).

50. Karimi, M., Inze, D. \& Depicker, A. GATEWAY vectors for Agrobacteriummediated plant transformation. Trends Plant Sci. 7, 193-195 (2002).

51. Nakagawa, T. et al. Development of series of gateway binary vectors, pGWBs, for realizing efficient construction of fusion genes for plant transformation. J. Biosci. Bioeng. 104, 34-41 (2007).

52. Suzaki, T. et al. Positive and negative regulation of cortical cell division during root nodule development in Lotus japonicus is accompanied by auxin response. Development 139, 3997-4006 (2012).

53. Kojima, M. et al. Highly sensitive and high-throughput analysis of plant hormones using MS-probe modification and liquid chromatography-tandem mass spectrometry: an application for hormone profiling in Oryza sativa. Plant Cell Physiol. 50, 1201-1214 (2009).

54. Takeda, N., Maekawa, T. \& Hayashi, M. Nuclear-localized and deregulated calcium- and calmodulin-dependent protein kinase activates rhizobial and mycorrhizal responses in Lotus japonicus. Plant Cell 24, 810-822 (2012).

\section{Acknowledgements}

We thank Satoru Okamoto (National Institute for Basic Biology (NIBB)) for providing the p35S-CLE-RS1, p35S-CLE-RS2 and p35S-GUS plasmids and Tsuyoshi Nakagawa (Shimane University) for providing the pGWB3 plasmid. We also thank Krzysztof Szczyglowskia (University of Western Ontario) for providing the hit1-1 mutant. We thank NIBB Model Plant Research Facility for providing facilities for the culture of plants and Sachiko Tanaka, Michiko Ichikawa and Yuko Ogawa (NIBB) for assisting with plan culture. This research was supported by MEXT/JSPS KAKENHI, Japan (25114519 to T.Su. and 25291066, 22128006 to M.Ka.).

\section{Author contributions}

T.Sa., T.Su., T.So. and M.Ka. designed the experiments. T.Sa., M.Ko. and H.S. performed experiments and analysed data. T.Sa., T.Su., T.So. and M.Ka. wrote the paper.

\section{Additional information}

Accession codes: The nucleotide sequences reported in this paper have been submitted to the NCBI database with accession numbers: LjIPT1, DQ436462; LjIPT2, DQ436463; LjIPT3, DQ436464; LjIPT4, DQ436465; LjIPT5, ABW77761; LjIPT9, EEE85226.2.

Supplementary Information accompanies this paper at http://www.nature.com/ naturecommunications

Competing financial interests: The authors declare no competing financial interests

Reprints and permission information is available online at http://npg.nature.com/ reprintsandpermissions/

How to cite this article: Sasaki, T. et al. Shoot-derived cytokinins systemically regulate root nodulation. Nat. Commun. 5:4983 doi: 10.1038/ncomms5983 (2014). 